\title{
Achievements and Challenges in Molecular Conductors
}

\section{Hidetoshi Fukuyama}

Tokyo University of Science, 1-3 Kagurazaka, Shinjuku-ku, Tokyo 162-8601, Japan; E-Mail: fukuyama@rs.kagu.tus.ac.jp; Tel.: +81-3-3260-4271; Fax: +81-3-3260-4772

Received: 1 April 2012; in revised form: 15 June 2012 / Accepted: 18 June 2012 /

Published: 5 July 2012

\begin{abstract}
Molecular solids are generally highly insulating. The creation of conducting molecular solids proved to be a major scientific challenge. As in the case of Si technology, the challenge started as impurity doping in band insulators and then developed into highly doped polymers, which are not crystalline. More conducting materials in crystalline forms have been realized in charge transfer (CT) complexes with two different kinds of molecules, where electrons are transferred between them in solids. In such CT complexes, not only conducting, but also even superconducting systems were achieved in 1980 and today more than 100 different superconductors are known. The most remarkable achievement in this direction was the realization of a truly metallic state in molecular solids based on a single kind of molecule. These are called single component molecular metals (SCMM) and consist of a rich variety of electronic properties. In these conducting molecular solids, CT and SCMM, many interesting electronic properties resulting from mutual Coulomb interactions and electron-phonon interactions have been explored so far, and these will be reviewed briefly in this article from a theoretical viewpoint. Challenges to come, based on these achievements, are also discussed at the end of this review.
\end{abstract}

Keywords: charge transfer salts; single component molecular metals; charge order; Mott insulator; dimer Mott; triangular lattice; spin liquid; massless Dirac electrons; tilted Weyl equation; $p$-d systems

\section{Introduction}

As has been most beautifully phrased by Anderson [1] "More is different", properties of solids consisting of a huge number of atoms and molecules are truly diverse and completely different from those of each isolated constituent. The most significant emergent features of solids are the clear 
distinction between metals and insulators, and the existence of phase transition such as ferromagnetism and superconductivity. Regarding the former, which is more fundamental, realization of the metallic state was not easy in general for solids consisting of molecules. The history of exploration of conducting molecular solids is, however, not so old. Nevertheless, achievements in the past 40 years are remarkable. Here it is to be noted that targets in realizing metallic states will be clearly identified by understanding another limiting factor, i.e., insulating states: band insulator (BI), Mott insulator (MI), charge ordered state (CO) and Anderson localized state. The former three are intrinsic; BI is due to the Pauli principle, while $\mathrm{MI}$ and $\mathrm{CO}$ are due to strong Coulomb interaction among electrons (strong correlation). The latter, Anderson localization, is due to disorder.

After some earlier efforts [2], semiconducting properties have been realized in $\mathrm{Br}$ doped perylenes [3]. The realization of conducting state here is through substitution of host by a small amount of elements with a different number of electrons in the outer shell, i.e., carrier doping into otherwise insulating host crystals (doped band insulators). This situation is similar to doped silicon. Doped polyacetylenes, which were realized in 1977 [4], belong to this family. More recently, carrier doping by external electric field (field-effect transistors, FET), which has long been familiar in two-dimensional electron gas in Si-MOS, has been pursued in search for proper materials for molecular devices [5]. Conducting states with rather high mobility has recently been realized, based on DNTT(dinaphtho[2,3-b:2',3'-f]thieno[3,2-b]thiophene) [6] and/or BTBT (2,7-Diphenyl[1]benzothieno[3,2-b]benzothiophene) [7] molecules. It is interesting to note that carrier doping even into MI has also been achieved recently [8]. In FET, carrier density is usually small and effects of disorder are inevitable, and then resistivity quite often builds up as the temperature is lowered. Eventually, the insulating state is realized as ground state, which is Anderson localized state. The processes toward such insulating states and their electronic properties have been studied extensively both experimentally and theoretically [9].

\section{Metallic Molecular Solids of Charge Transfer Type}

\subsection{TTF-TCNQ}

More conducting states than those due to doping have been realized around 1970 in solids with two different kinds of molecules, where electrons are transferred from one kind of molecule to another, called charge transfer (CT) salts. Here, two kinds of molecules work as donors (D) and acceptors (A), respectively. With a partial amount of electron per molecule transferred between $\mathrm{D}$ and $\mathrm{A}$, conducting carriers are created. In the case of TTF-TCNQ (tetrathiafulvalene-tetracyanoquinodemethane), which is very one-dimensional and has been studied in detail, the carrier number per molecule is around 0.3, and both electrons and holes are present (semi-metallic). In this system, conductivity is anomalously enhanced around $50 \mathrm{~K}$ as the temperature is lowered [10]; a fact which stimulated many investigations [11,12]. The ground state in this case insulates due to the Peierls distortion resulting in superstructure, and then this insulating state is understood as BI in the new periodic structure. Tt has since been theoretically clarified [13] that one-dimensional electron systems generally display instability which is associated with the Fermi surface, e.g., charge density wave (CDW) and spin 
density wave (SDW), leading to the insulating ground state. The case of TTF-TCNQ is a good example of CDW.

\section{2. $(\mathrm{TMTCF})_{2} \mathrm{X}$}

The molecule, TMTCF, stands for either TMTSF (tetramethyltetraselenafulvalene) or TMTTF (tetramethyltetrathiafulvalene) with $\mathrm{C}$ representing a chalcogen. (TMTCF) ${ }_{2} X$ is $\mathrm{CT}$ salt with the composition of $\mathrm{D}_{2} \mathrm{~A}$. Here an acceptor $(\mathrm{A})$ such as $\mathrm{PF}_{6}$ has strong potential to attract an electron to be a closed shell such as $\mathrm{A}^{-}$. As a function of pressure, either external or chemical (by substituting TMTSF by TMTTF molecule, or changing anions $X$ ), this family shows a very rich phase diagram, which includes superconductivity (SC), SDW, antiferromagnetism (AF), spin-Peierls (SP) and charge ordered (CO) phases [14]. The first superconducting state in molecular solids was realized in one such family member: (TMTSF) ${ }_{2} \mathrm{PF}_{6},[15,16]$ in 1980. (More than 100 superconducting molecular solids are now known [17].) The latest understanding of the phase diagram of this family is depicted in Figure $1[18,19]$. Note that the relationship between AF and SP is seen to be intricate [20].

Figure 1. Phase diagram of (TMTCF $)_{2} X$ family on the plane of pressure (either external or chemical) and temperature based on References 18 and 19. SC: superconductor; IC-SDW: incommensurate spin density wave; AF: antifrromagnetism; SP: spin-Peierls; FCO: ferroelectric charge order; $\mathrm{CL}$ : charge localization; $T_{\mathrm{\rho}}$ : characteristic temperature at resistivity minima.

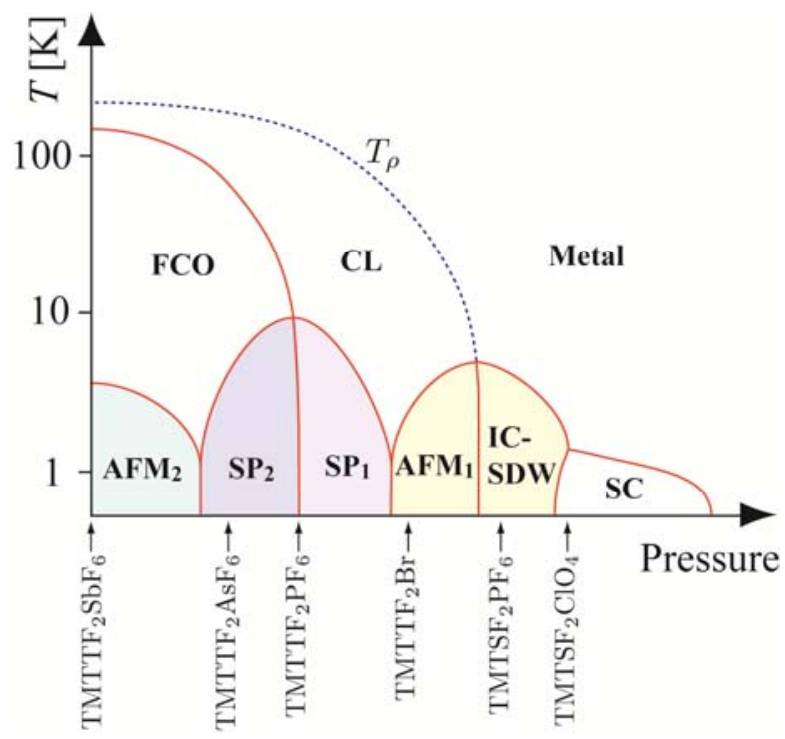

Band structure caused some confusion in the early stages, but eventually the validity of tight-binding approximation based on molecular orbitals, HOMO (highest occupied molecular orbital) and LUMO (lowest unoccupied molecular orbital) [21,22], was demonstrated [23]. With this 2:1 composition, the Bloch band consisting of HOMO of TMTCF molecules will be 3/4-filled (1/4 hole-filling). The actual band structure of (TMTCF $)_{2} X$ is very one-dimensional, reflecting chain structures but with non-negligible inter-chain transfer integrals and interactions. This explains the diversity seen in the phase diagram together with various interesting phenomena under magnetic field [24,25]. 
There have been issues raised associated with the phase diagram (Figure 1), especially associated with $T_{\rho}$. In order to understand the background of these issues, it is important to note that two limiting states are expected as ground states in 1/4-filling in the presence of strong Coulomb interactions; charge order (CO) in cases without dimerization [26], and Mott insulators in the presence of strong dimerization (known as dimer Mott insulator; DM) as schematically shown in Figure 2. In TMTCF, there is a finite dimerization of the order of $10 \%$ along the chain because of its crystal structure, and then values of transfer integrals alternate. A question then arises: should (TMTCF) ${ }_{2} X$ be considered as half-full because of finite dimerization? If this aspect is emphasized, Mott physics will play crucial roles [27,28]. However, it has been indicated by simple calculations based on mean-field approximation for Coulomb interaction, together with finite dimerization in this $1 / 4$-filling case, that $\mathrm{CO}$ is possible [29]. In parallel to, and independent of this theoretical indication, $\mathrm{CO}$ was observed by NMR [30] in another quasi-one-dimensional CT, (DI-DCNQI) $2 \mathrm{Ag}$ (DI-DCNQI $=2,5$-diiodo- $N, N^{\prime}$-Dicyanobenzoquinonediimine2,5-diiodo- $N, N^{\prime}$-Dicyanobenzoquinonediimine), which is without dimerization at high temperatures. (Once CO sets in, a particular type of dimerization is stabilized [31,32].) A little later CO was also identified by NMR in an experiment with (TMTTF) ${ }_{2} X$ $\left(X=\mathrm{PF}_{6}\right.$ and $\left.\mathrm{AsF}_{6}\right)$ with finite dimerization [33]. Moreover anomalies and/or phase transition type critical behaviors in dielectric constant, associated with $\mathrm{CO}$, have been identified in (TMTTF) ${ }_{2} X$ $\left(X=\mathrm{PF}_{6}, \mathrm{AsF}_{6}, \mathrm{SbF}_{6}\right)$ [34-36], which resolved the long-standing mystery of existence of "structureless transition" [37]. This indicates that the essence of electronic states discriminating between $1 / 2$-filling and 1/4-filling is quantitative [38]. At the same time, this finding has clarified that not only mutual Coulomb interaction, but also electron-phonon interactions [20,39], play important roles in accordance with the existence of spin-Peierls phase next to anitiferromagnetic state on one hand, and various patterns of charge ordering $[39,40]$ not simply expected by Coulomb interaction alone, on the other hand. Moreover, it is worth noting in Figure 1 that there are two different kinds of spin-Peierls (SP) and antiferromagnetic (AF) phases as a function of pressure. SP1 and AF1 had been understood in terms of the increase of dimensionality as pressure increases [41], while SP2 and AF2, which are apparently associated with $\mathrm{CO}$ [42-45], are now being proposed as resulting from the interplay between mutual Coulomb interactions within a chain and between chains, electron phonon interaction and electron hopping between chains [19].

Figure 2. Two limiting cases of 1/4-filled bands: (a) Charge ordered states in the absence of dimerization; (b) Mott insulators in the presence of strong dimerization.
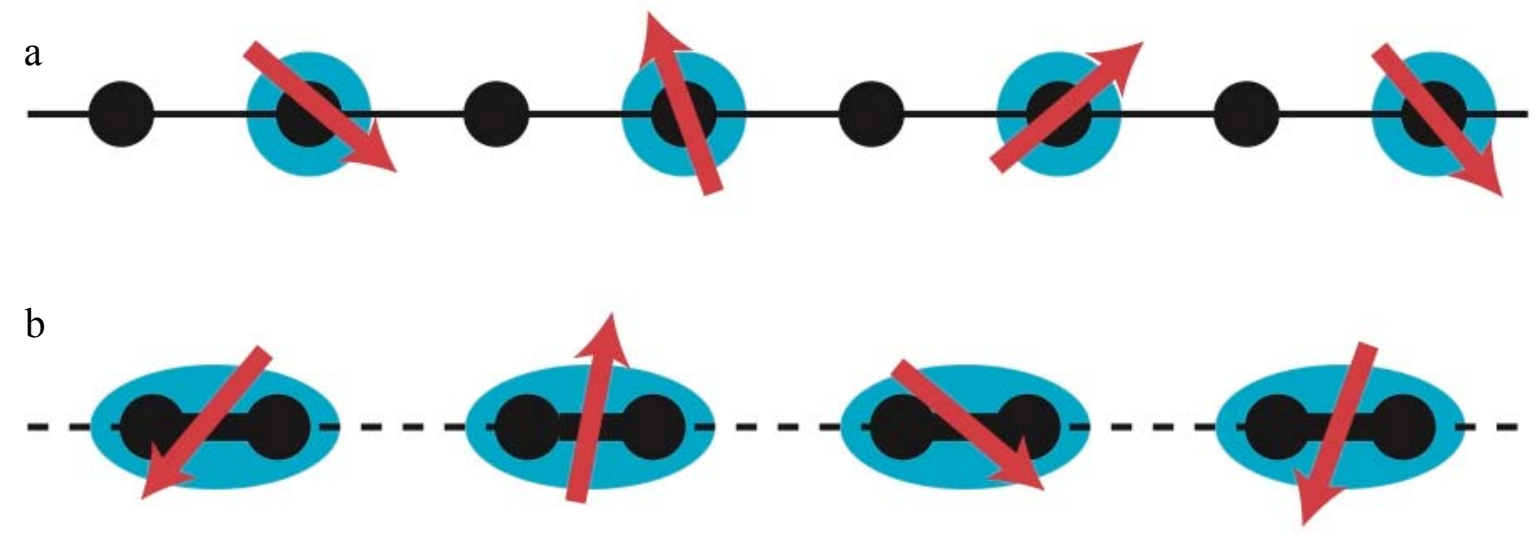


\section{3. $(E T)_{2} X$}

$\mathrm{CT}$ salts with the same composition ratio, $\mathrm{D}_{2} \mathrm{~A}$, and with BEDT-TTF (bis(ethylenedithio)tetra-thia-fulvalence, abbreviated as ET) molecules as donors, have been studied in great detail [46-48]. (ET) ${ }_{2} X$ compounds have conducting ET layers separated by an insulating one consisting of acceptors. Because of the crystal structure, electronic states are very two-dimensional in contrast to (TMTCF $)_{2} X$. There is diversity in the spatial location of ET molecules within a unit cell, called poly-types, each of which shows characteristic electronic properties. It had proven difficult to underpin the crucial parameters leading to such diversity in spite of the fact that the carrier density is the same: 1/4-hole-filled. The essence of the diversity of electronic properties, theoretically, is that the basis is the band structure derived by the tight-binding approximation for the transfer integrals, $t_{i j}$. Effects of mutual Coulomb interaction are studied based on the same molecular orbitals in terms of parameters such as " $U$ " in the Hubbard model, but also with spatially extended ones between neighboring orbitals, $V_{i j}$ (especially those between nearest neighbors, $V$ ). The canonical model in such circumstances, which may be termed as "extended Hubbard model", is given as follows:

$$
\mathrm{H}=\sum_{<i j>} \sum_{\sigma}\left(t_{i j} c_{i \sigma}^{\dagger} c_{j \sigma}+\text { h.c. }\right)+\sum_{i} U n_{i \uparrow} \mathrm{n}_{\mathrm{i} \downarrow}+\sum_{<i j>} V_{i j} n_{i} n_{j}
$$

It is important to note that values of $U$ and $V$ (especially the former) are more or less the same in all (ET) ${ }_{2} X$ and features particular to each polytype are due to the transfer integrals, $t_{i j}$. It turned out that, from the values of electron transfer integrals, there are different degrees of dimerization depending on the polytypes. As emphasized in Figure 2, two limiting states are expected in 1/4-filling depending on the degree of dimerization, CO and DM, though in reality it is usually somewhat in between. Moreover, crystal structures (values of transfer integrals, to be precise) are close to triangular lattice of either single ET molecule or dimers as a unit (in cases with strong dimerization) as shown in Figure 3, where the crucial parameter is $t^{\prime} / t$. Hence the degree of dimerization and proximity to triangular lattices have turned out to be the key parameters to classify the whole family of $(\mathrm{ET})_{2} X$. Among scientific highlights associated with these $(\mathrm{ET})_{2} X$ compounds, are the realization of spin liquid in triangular $\kappa-(\mathrm{ET})_{2}$ $\mathrm{Cu}_{2}(\mathrm{CN})_{3}$, and discovery of massless Dirac electrons in $\alpha-(\mathrm{ET})_{2} \mathrm{I}_{3}$ with $\mathrm{CO}$ (charge disproportionation to be precise); both of which are introduced in the following.

Figure 3. Transfer integrals in triangular lattices, $t$ and $t^{\prime}$.

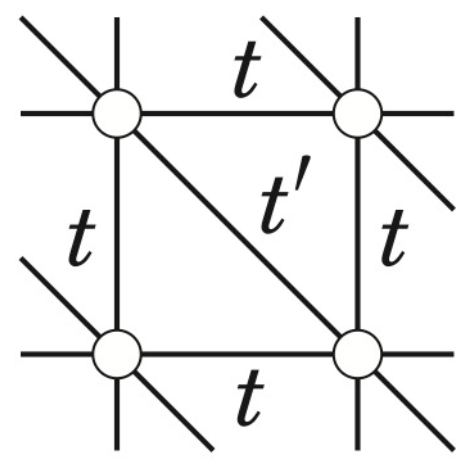




\subsubsection{Spin Liquid State in $\kappa-(\mathrm{ET})_{2} \mathrm{Cu}_{2}(\mathrm{CN})_{3}$}

In $\kappa$-polytype $(\mathrm{ET})_{2} X$, which forms an interesting phase diagram with insulating and superconducting states next to each other on the plane of effective pressure and temperature $[49,50]$, there is strong dimerization and then the electronic state is identified by treating a dimer as a unit. Each dimer has one hole and then Mott insulators (dimer Mott) are expected to form if Coulomb interactions are strong. The resultant lattice structures of dimers are triangular $[46,51]$ but with small deviations from perfect triangular lattice depending on $X$, which is characterized by $t^{\prime} / t$. In the present case of dimer Mott on triangular lattice, spin 1/2 exists on each lattice as shown in Figure 4a among which superexchange interactions, $J$ and $J^{\prime}$, associated with transfer integrals $t$ and $t^{\prime}$, are operating. Comparing cases with $X_{1}=\mathrm{Cu}_{2}(\mathrm{CN})_{3}\left(t^{\prime} / t=1.05\right)$ and with $X_{2}=\mathrm{Cu}\left[\mathrm{N}(\mathrm{CN})_{2}\right] \mathrm{Cl}\left(t^{\prime} / t=0.74\right)$, it is seen from temperature dependences of susceptibility [52] that there is a magnetic transition for $X_{2}$ but not for $X_{1}$. Actually there is no sign of magnetic transition in case of $X_{1}$ down to $32 \mathrm{mK}$ which is of the order of $\mathrm{J} / 10^{4}$ with $J \sim 250 \mathrm{~K}$. This is the first experimental realization of spin liquid which was proposed by Anderson in 1973 [53]. Since then, various candidates of spin liquid states have been reported including another kind of molecular solid, $\beta^{\prime}-X \quad\left[\mathrm{Pd} \quad(\mathrm{dmit})_{2}\right]_{2}$ (dmit $=1,3$-dithiol-2-thione-4,5-dithiolate) with $X=\mathrm{EtMe}_{3} \mathrm{Sb}$ [54,55]. It has been clarified that this family has various ground states as a function of $t^{\prime} / t$, including antiferromagnetic long range order, charge order and spin liquid state in between [56]. Specific heat, NMR relaxation time and thermal conductivity in these candidate materials are reported to have puzzling low temperature behaviors which appear not to be consistent with the framework of conventional understanding of spin systems, for which various explanations have been proposed [57]. It is interesting to note that a very small interaction (much smaller than $J$ ) will manifest itself toward absolute zero in real materials, leading to some kind of ordering, mainly in the short-range, as in the case of weakly coupled chain systems. Hence, it is possible that eventually interaction paths heading toward absolute zero become one-dimensional, as in percolation processes.

Figure 4. Frustrated lattices in (a) spins (arrows) and (b) charges (shaded circles).

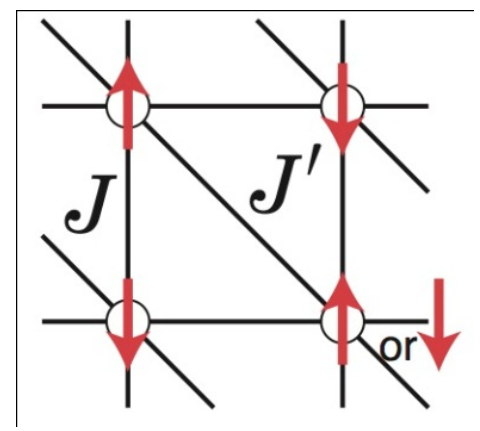

(a)

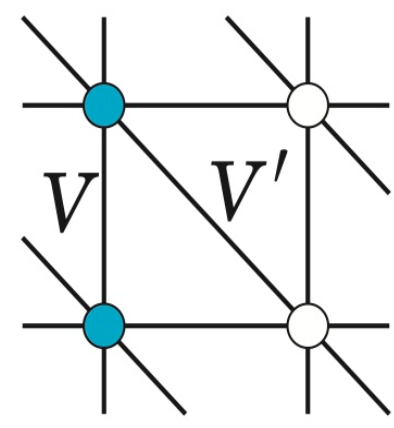

(b)

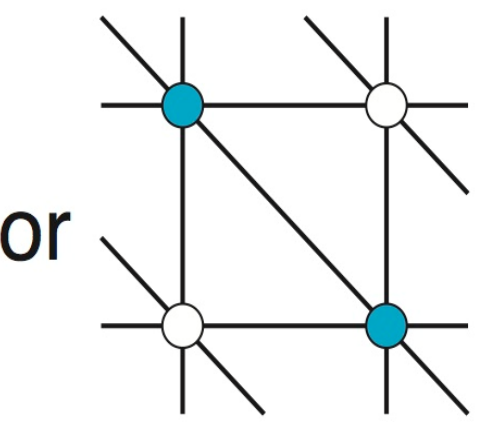

One recent surprise associated with $\kappa-(\mathrm{ET})_{2} \mathrm{Cu}_{2}(\mathrm{CN})_{3}$, is the finding of strong frequency dependences of dielectric constant [58] indicating the presence of active charge degree of freedom which is beyond the concept of dimer Mott insulator. There are proposals [59-62] which point to the proximity of dimer Mott in $\kappa$-polytype to charge ordered state. In this context, it interesting to note that in $\beta^{\prime}-X$ $\left[\mathrm{Pd}(\mathrm{dmit})_{2}\right]_{2}$, spin liquid is realized for $X=\mathrm{EtMe}_{3} \mathrm{Sb}\left(t^{\prime} / t=0.92\right)$, while charge ordering occurs for 
$X=\mathrm{Et} 2 \mathrm{Me}_{2} \mathrm{Sb}\left(t^{\prime} / t=1.01\right)$, indicating spin liquid state here is also in the proximity of charge ordering. Another interesting experimental indication is that this anomalous dielectric property may depend on whether the system is either spin liquid or magnetic [63]. This would reflect the subtle interplay between spin and charge sectors, which deserves further detailed studies both experimentally and theoretically.

\subsubsection{Charge Glass State in $\theta$-polytype}

The spin liquid state, which is a unique state of matter, is due to the frustration of quantum spins. A similar situation can be imagined for the spatial arrangement of electronic charges for the present 1/4-filled cases, as schematically shown in Figure 4b. With such frustrations, charges will not order, even under strong mutual interactions as suggested theoretically [64]. Such a state may be called charge glass. In $\theta$-polytype of $\mathrm{ET}_{2} \mathrm{X}$, which displays an interesting phase diagram with superconductivity and charge order on the plane of temperature and dihedral angle between neighboring molecules [17], many experimental results including X-ray and Raman scattering, NMR and non-linear transport in the metallic states indicate the existence of short range charge order [65]. It is challenging to search for possible superconducting states in such charge glass states, since the energy scale governing the critical temperature can be large in liquid.

\subsubsection{Massless Dirac Electrons in $\alpha-(\mathrm{ET})_{2} \mathrm{I}_{3}$}

In $\alpha-(E T)_{2} I_{3}$ there is no clear dimerization and the unit cell has four ET molecules. At ambient pressure, there is metal-insulator transition at around $T_{\mathrm{MI}}=135 \mathrm{~K}$ [66] which is now understood as due to charge ordering $[47,67]$. The $\mathrm{T}_{\mathrm{MI}}$ is reduced by external pressure and eventually disappears above $15 \mathrm{Kbar}$. The resistivity of the metallic state is very particular and almost independent of temperature. Measurement of Hall mobility disclosed that the effective carrier number is reduced by several orders of magnitude between room temperature and liquid He temperature. This implies that mobility is enhanced by the same amount in the same temperature range. Very careful theoretical analysis of band structure under such pressure, based on tight-binding approximation, disclosed [68,69] that the electronic band structure has a very particular feature of having accidental degeneracy (crossing point) off the symmetry point (two crossing points in the Brillouin zone) and that the Fermi energy is located precisely at this crossing due to 3/4-filling). This fact has been confirmed later by first principle band calculations [70,71]. The effective Hamiltonian around each of these crossing points is given by the tilted Weyl equation [72] given as follows,

$$
H=\sum_{\rho=0,1,2,3} k \cdot v_{\rho} \sigma_{\rho}
$$

where $\sigma_{0}=1, \sigma_{\rho}(\alpha=1,2,3)$ represents the Pauli matrix and $v_{\rho}$ is velocity. This leads to massless Dirac electrons as in graphite [73,74] and graphene [75] but with finite tilting in the present case, which reflects the fact that the crossing points are located away from the symmetry points in the Brillouin zone. The essential difference of the materials, is that graphene is just one layer but $\alpha-(\mathrm{ET})_{2} \mathrm{I}_{3}$ is a bulk crystal. Many particular features of electronic properties are expected for such massless Dirac electrons, including transport, especially magneto-transport, properties both within and between layers. This is due to Landau quantization by magnetic field applied perpendicularly to the layers, which is 
different from ordinary quantization as seen in Figure 5 [73]; there is always a Landau level at the crossing energy called 0-th level. This is due to essential coupling between two bands in terms of magnetic field, i.e., inter-band effects of magnetic field [76]. The energy level quantization by magnetic field, shown in Figure 5, leads to a new type of quantum Hall effect as has been confirmed experimentally in graphenes [75]. The non-monotonic field dependences of magneto-resistance between the layers in $\alpha$-(ET) $)_{2} \mathrm{I}_{3}$ have been shown to be consistent with the existence of this 0 -th level [77]. Responses of massless Dirac electrons to external magnetic field have particular features not only in such high magnetic fields, but also in weak fields as has been known for many years for the case of orbital susceptibility $\chi$, which has large contributions only when the chemical potential is close to the crossing energy [78]. On the other hand, behaviors of the Hall conductivity $\sigma_{x y}$ have been clarified only recently, first without [79] and then with, tilting [80]. The results of dependences on chemical potential $(\mu)$ of $\chi$ and $\sigma_{x y}$ [79] together with those of well-studied $\sigma_{x x}$ [81] under the assumption of constant damping, $\Gamma$, are shown in Figure 6 . The existence of finite tilting affects the results in Figure 6 quantitatively, but qualitative features are unchanged. It is important to note that $\sigma_{x y}$ vanishes at the crossing energy, which is very natural from the viewpoint of electron-hole symmetry. However, this has important implications, since the Hall coefficient $R=\sigma_{x y} / H \sigma_{x x}{ }^{2}$ should vanish if the chemical potential is at the crossing energy. This is in sharp contrast to conventional understanding that $R$ is proportional to the inverse of the effective carrier density and then it would diverge toward the crossing energy, either negatively (electron-like) or positively (hole-like), as shown by broken lines in Figure 6d. This is a very illuminating example of the essential importance of the inter-band effects of magnetic fields.

Figure 5. Magnetic field dependences of energy levels of two-dimensional massless Dirac electrons with perpendicular field.

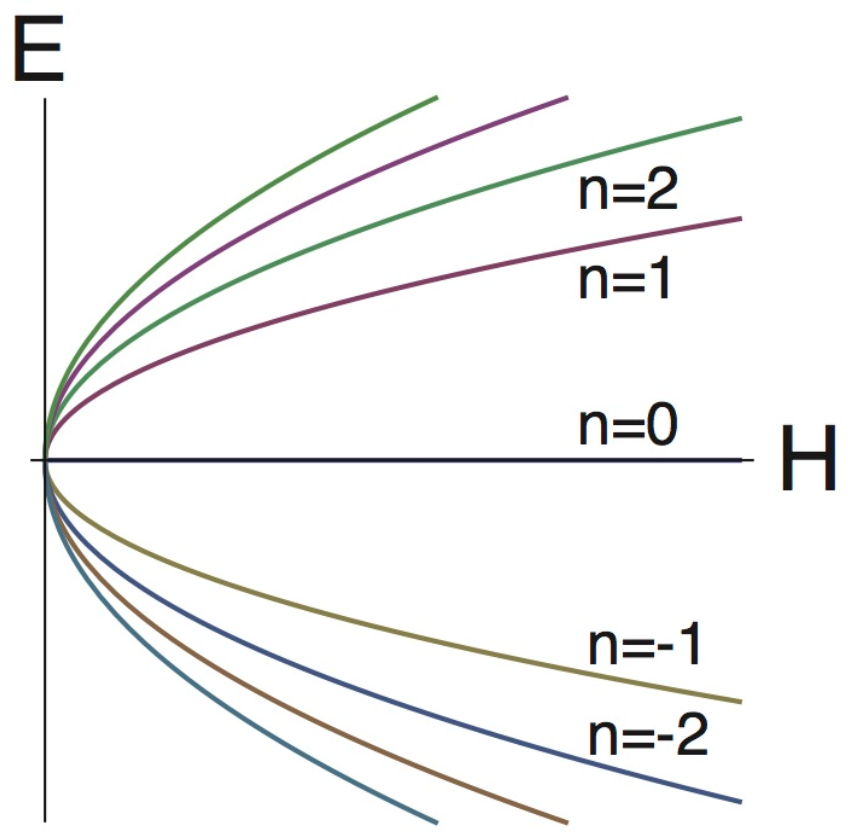


Figure 6. Chemical potential $(\mu)$ dependences of: (a) orbital susceptibility, $\chi$; (b) diagonal conductivity, $\sigma_{x x}$; (c) Hall conductivity, $\sigma_{x y}$; and (d) Hall coefficient, $R$, respectively, based on Reference 79. Here $X=\mu / \Gamma, \Gamma$ being the damping constant, $\chi_{0}=\mathrm{e}^{2} v^{2} / 3 \pi^{2} c^{2} \Gamma$, $\sigma_{0}=\mathrm{e}^{2} / 2 \pi^{2}$, and $\sigma_{x y}^{0}=\mathrm{e}^{3} v^{2} H / 4 \pi^{2} c \Gamma$, respectively. The broken lines in Figure $6 \mathrm{~d}$ for Hall coefficient correspond to the inverse of carrier density of either electrons or holes.
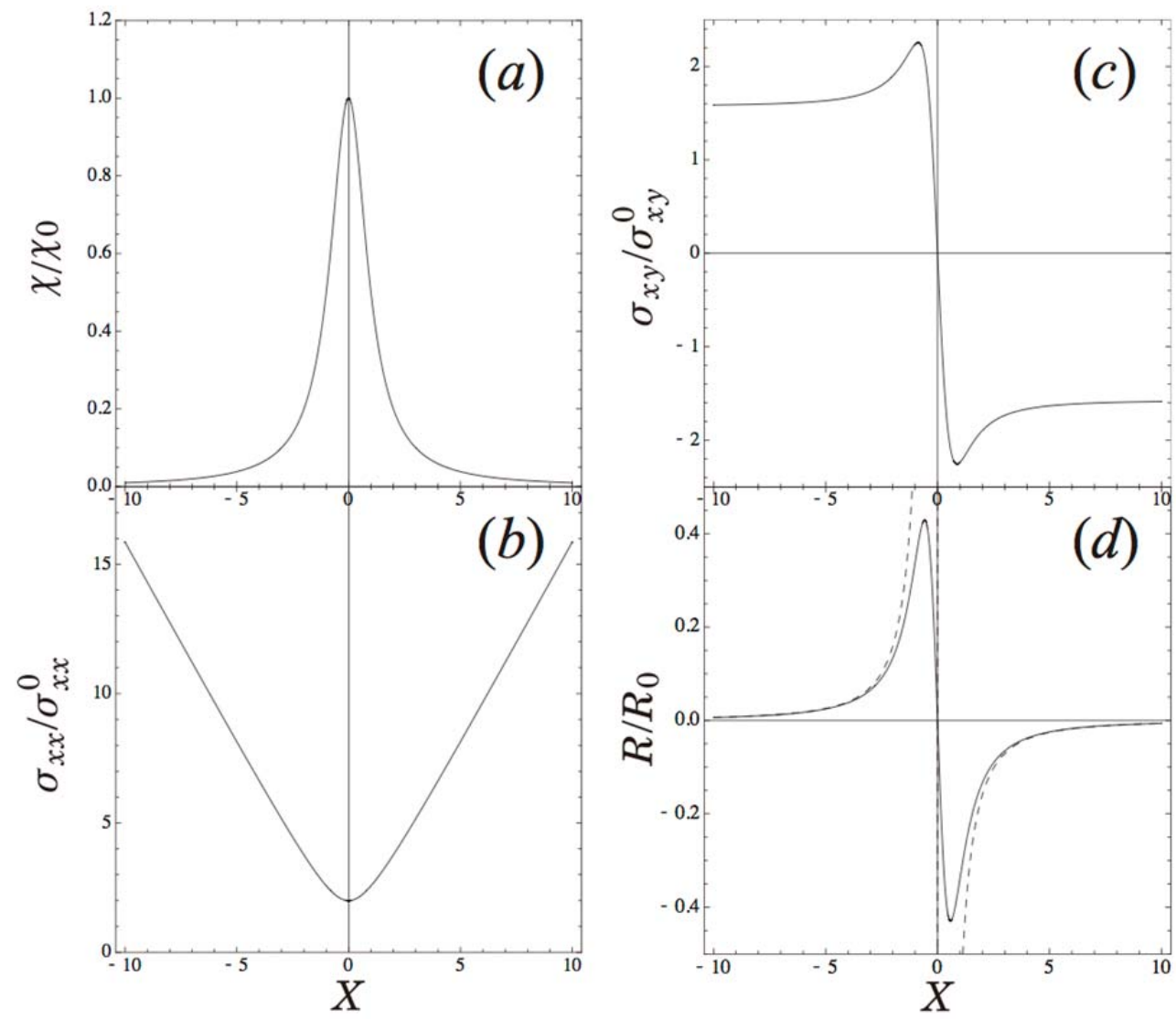

Experimentally, Hall coefficient $R$ in $\alpha-(E T)_{2} \mathrm{I}_{3}$ shows very interesting temperature dependences as seen in Figure 7 [82], which discloses a very abrupt but continuous change. This is proposed [80] to be due to temperature dependence of chemical potential resulting from asymmetry of energy dependences of density of states near the crossing energy [71] and possible existence of $\mathrm{I}_{3}$ defects. Further studies on this interesting possibility, both theoretically and experimentally, will disclose the nature of electronic states near the crossing points in more detail, which may be sensitive to interactions as suggested by recent NMR experiments in [83] as well as theoretically in [84]. Regarding weak temperature dependences of resistivity, together with very strong temperature dependences of effective mobility and carrier density, no detailed studies have yet been carried out. We may, however, argue as follows based on the results obtained in Figure 6 at absolute zero, which shows conductivity almost linear in energy compared with almost no temperature dependences in experiments. This will be resolved if $\Gamma$ is proportional to temperature. Then this, from the weak dependences on energy of $\sigma_{x y}$ in Figure 6, will lead to $\sigma_{x y} \sim 1 / \Gamma^{2}$, which predicts $R$ is proportional to $1 / \Gamma^{2}$, which is in qualitative agreement with experiments, though detailed analysis based on solid calculations is definitely needed. 
Figure 7. Experimental results of Hall coefficient, $R$, of $\alpha-(\mathrm{ET})_{2} \mathrm{I}_{3}$ as a function of temperature [82]. Note that $R$ in electron-doped cases changes sign very sharply at some characteristic temperature which depends on samples, whereas the sign of $R$ in hole-doped cases is unchanged.

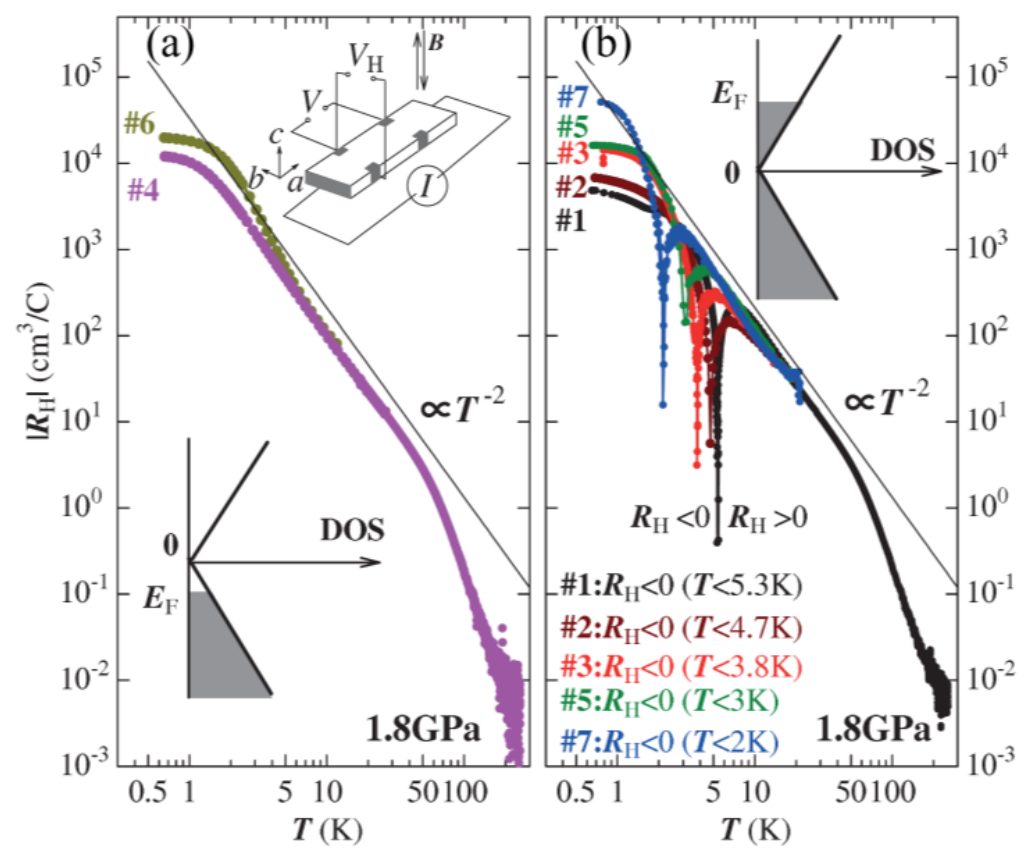

\section{Charge Transfer Complexes with d Electrons}

There are cases where a molecule contains a metallic ion, quite often transition metals with d-orbitals. These cases will be classified into two groups depending on the degree of localization of d-states, either (A) localized spins (S) or (B) fluctuating valences. The former, (A), includes $\lambda$-(BETS) $\left(\mathrm{FeCl}_{4}\right)_{2}$ (BETS $=$ bis(ethylenedithio)tetraselenafulvalene) with $\mathrm{S}=5 / 2$ associated with high spin state of $\mathrm{Fe}^{+3}$ being a surprising example of superconductivity induced by magnetic field, applied parallel to the layer $[85,86]$ and TTP $\left[\mathrm{FePc}(\mathrm{CN})_{2}\right]_{2}(\mathrm{TTP}=$ tetraphenylphosphonium, Pc = phthalocyaninato $)$ with $\mathrm{S}=1 / 2$ due to low spin state of $\mathrm{Fe}^{3+}$, showing strong magneto-resistance [87], while the latter, (B), includes $\mathrm{V}(\mathrm{TCNE}) x$ (TCNE = tetracyanoethylene), which is an example of a room-temperature ferromagnet [88-90]. The case of $\mathrm{DCNQI}_{2} \mathrm{Cu}$ (DCNQI $=2,5$-Disubstituted $N, N$-Dicyanobenzoquinonediimines), which shows dramatic metal-insulator transition as a function of temperature and chemical pressure, can be considered to be a very special example of valence fluctuation in metallic state and localized spins in insulating states tuned by temperature [91,92]. Such features of mixed characteristics are also found in single component molecular metals, as will be discussed in the following.

\section{Single Component Molecular Metals}

In 2001, metallic states based on a single kind of molecule, Ni(tmdt) $(\mathrm{tmdt}=$ trimethylenetetrathiafulvalenedithiolate), was realized. This is really an important step in the research history of conducting molecular solids [2,93,94]. As seen in Figure 8, the Ni atom in this molecule is located at the center coupled to a ligand (tmdt) on each side. The metallic state here is due 
to the overlap of HOMO and LUMO bands as designed (which is a surprising fact!). The existence of Fermi surfaces has been experimentally confirmed by dHvA [95] whose oscillations are in agreement with results of band structure calculations, first by tight-binding approximation [93] and later by more detailed first principle calculations [96-98] agreeing with each other. It is to be noted that a large positive and isotropic magneto-resistance has been observed in this system [99], whose origin is not identified. Later SCMM, based on another kind of molecule, Au(tmdt) ${ }_{2}$, has been synthesized [100] which shows the onset of anti-ferromagnetism at around $110 \mathrm{~K}$ with a relatively large magnetic moment [101,102] and metallic state maintained at low temperature. The nature of this antiferromagnetic state has been studied, based on first principle band calculations [97,98] and on multiband Hubbard model based on the numerical fitting to band calculations [103]. However, theoretical values for the magnetic moment are clearly smaller compared with experimental values [102], whose origin has not yet been understood. More recently another SCMM based on $\mathrm{Cu}(\mathrm{tmdt})_{2}$ has been synthesized [104]. In contrast to the preceding examples, this system with $\mathrm{Cu}$ insulates below room temperature and the magnetic susceptibility indicates the existence of a localized magnetic moment associated with $\mathrm{Cu}^{2+}$. Exploration of the nature of anti-ferromagnetism in SCMM with $\mathrm{Au}$ and $\mathrm{Cu}$ ions by identifying relative roles of d-orbitals, is an interesting subject. If SCMM with other transition metal elements, such as Fe and Mn, is realized, the scope of research of this family in material science would be greatly widened.

Figure 8. Schematic representation of the molecule, $\mathrm{Ni}(\mathrm{tmdt})_{2}$, which led to the first single component molecular metal.

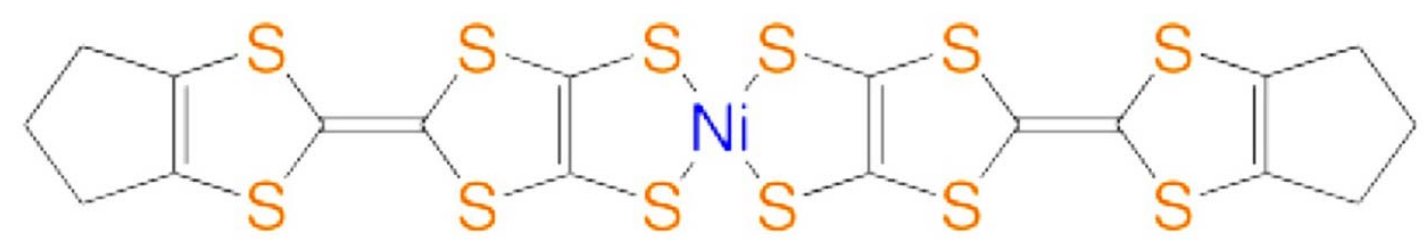

\section{Challenges: Electronic States in Bio-material}

As seen in the previous sections, understanding properties of molecular solids based on electronic states has experienced remarkable changes over the past 40 years. This is due to the progress of various experimental techniques on one hand, and theoretical analysis with models based on tight-biding approximation on the other hand. It will be natural to expect that research targets of condensed matter science gradually include non-crystalline molecular systems, e.g. bio-material such as proteins and DNA. In metalloproteins, for example, local structures around metallic ions are similar to those discussed in Sections 3 and 4, especially the latter. The non-crystalline features in these bio-materials might appear to be a difficult barrier to overcome from the view of solid state science. This is not the case in essence, however. This is due to the validity of tight-binding approximation for electronic states in molecular systems. Actually, there is already pioneering work [105] trying to search for key parameters in the tight-binding approximation between two amino-acids targeting construction of electronic states of proteins based on amino acids. This new framework has been applied recently to electron transfer in proteins [106]. Likewise, theoretical efforts are under way to identify electronic states and then functionalities of myoglobin stimulated by the state of the art 
experiment [107]. It has been indicated in this experiment that, for both reduced $\left(\mathrm{Fe}^{2+}\right)$ and oxidized $\left(\mathrm{Fe}^{3+}\right)$ states, the removal or addition of an electron primarily involves charge changes on ligand sites, and not the Fe-sites, which is in accordance with results based on optical spectra many years ago $[108,109]$. In order to carry out systematic researches in this direction, coordinated activities are needed among sample preparation, measurement and theoretical analysis, through collaboration between condensed-matter physics, chemistry and biology [110,111].

\section{Conclusion}

Efforts and achievements in the past 40 years in physics and chemistry concerning the science of conducting molecular solids, have been briefly reviewed, starting form charge transfer salts of $\mathrm{D}_{2} \mathrm{~A}$, such as (TMTTF $)_{2} X$ and (ET) $)_{2} X$, to single component molecular metals (SCMM). In the former which are 1/4-filled systems, it has been indicated that charge disproportionation and charge ordering are frequently realized. In the $\mathrm{ET}_{2} X$ family, two kinds of remarkable states of matter have been realized - the spin liquid, which was predicted by Anderson in 1973; and massless Dirac electrons described by the tilted Weyl equation - and each show very particular electronic properties. It has been pointed out also that when d-electrons couple with conducting $\mathrm{p}$ electrons, i.e., $\mathrm{p}$-d systems, there are two distinct states, depending on the degree of localization of $\mathrm{d}$ electrons; localized spins or valence-fluctuating. In SCMM, the constituting molecule has a metallic ion at the center linked to ligands on each side. In the first example of SCMM, Ni(tmdt) $)_{2}$, electrons are transferred to ligands and no appreciable coupling between $d$ and $p$ electrons occurs, whereas in later examples, $\mathrm{Au}(\mathrm{tmdt})_{2}$ and $\mathrm{Cu}(\mathrm{tmdt})_{2}$, $\mathrm{d}$ electrons of metallic ions play important roles leading to different types of magnetism. Such particular spatial coordination between d elements and ligands is commonly seen in bio-materials, especially in metalloproteins, which will be interesting and important research targets in the very near future.

\section{Acknowledgments}

The author thanks many colleagues both in theory and experiment for very informative discussions in various stages. Especially illuminating discussions with Hayao and Akiko Kobayashi, Kiyoyuki Terakura, Toshihiro Takahashi, Yoshikazu Suzumura, Reizo Kato, Kazushi Kanoda, Shoji Ishibashi, Hiori Kino, Hitoshi Seo, Chisa Hotta and Akito Kobayashhi are greatly acknowledged. Comments on the draft by Reizo Kato and helps by Hitoshi Seo, and Ken Yokoyama for preparing figures are appreciated. Special thanks are due to Ms. Kurakata for her kind help for preparing the manuscript.

\section{References}

1. Anderson, P.W. More is different. Science 1972, 177, 393-396.

2. Kobayashi, A.; Okano, Y.; Kobayashi, H. Molecular design and physical properties of single-component molecular metals. J. Phys. Soc. Jpn. 2006, 75, 051002:1-051002:12.

3. Akamatsu, H.; Inokuchi, H.; Matsunaga, Y. Electrical conductivity of the perylene-bromine complex. Nature 1954, 173, 168-169. 
4. Chiang, C.K.; Fincher, C.R., Jr.; Park, Y.W.; Heeger, A.J.; Shirakawa, H.; Louis, E.J.; Gau, S.C.; MacDiarmid, A.G. Electrical conductivity in doped polyacetylene. Phys. Rev. Lett. 1977, 39, 1098-1101.

5. Bredas, J.L.; Beljonne, D.; Coropceanu, V.; Cornil, J. Charge-transfer and energy-transfer processes in $\pi$-conjugated oligomers and polymers: A molecular picture. Chem. Rev. 2004, 104, 4971-5004.

6. Yamamoto, T.; Takimiya, K. Facile synthesis of highly $\pi$-extended heteroarenes, dinaphtho[2,3-b:2',3'-f]chalcogenopheno[3,2-b]chalcogenophenes, and their application to field-effect transistors. J. Am. Chem. Soc. 2007, 129, 2224-2225.

7. Takimiya, K.; Ebata, H.; Sakamoto, K.; Izawa, T.; Otsubo, T.; Kunugi, Y. 2,7-Diphenyl[1]benzothieno[3,2-b]benzothiophene, a new organic semiconductor for air-stable organic field-effect transistors with mobilities up to $2.0 \mathrm{~cm}^{2} \mathrm{~V}^{-1} \mathrm{~s}^{-1} . J$. Am. Chem. Soc. 2006, 128, 12604-12605.

8. Kawasugi, Y.; Yamamoto, H.M.; Tajima, N.; Fukunaga, T.; Tsukagoshi, K.; Kato, R. Field-induced carrier delocalization in the strain-induced Mott insulating state of an organic superconductor. Phys. Rev. Lett. 2009, 103, 116801:1-116801:4.

9. Nagaoka, Y. Theory of Anderson localization-A historical survey. Prog. Theor. Phys. Suppl. 1985, 84, 1-15.

10. Coleman, L.B.; Cohen, M.J.; Sandman, D.J.; Yamagishi, F.G.; Garito, A.F.; Heeger, A.J. Superconducting fluctuations and the peierls instability in an organic solid. Solid State Commun. 1973, 12, 1125-1132.

11. Devreese, J.T.; Evrard, R.P.; van Doren, V.E. Highly Conducting One-Dimensional Solids; Plenum Press: New York, NY, USA, 1979.

12. Jerome, D. Organic conductors: From charge density wave TTF-TCNQ to superconducting (TMTSF) ${ }_{2} \mathrm{PF}_{6}$. Chem. Rev. 2004, 104, 5565-5592.

13. Solyom, J. The Fermi gas model of one-dimensional conductors. Adv. Phys. 1979, 28, 201-303.

14. Brown, S.E.; Chaikin, P.M.; Naughton, M.J. La Tour des Sels de Bechgaard. In The Physics of Organic Superconductors and Conductors; Lebed, A., Ed.; Spring Springer Series in Materials: Berlin/Heidelberg, Geramny, 2008; pp. 357-512.

15. Jerome, D.; Mazaud, A.; Ribault, M.; Bechgaard, K. Superconductivity in a synthetic organic conductor (TMTSF) ${ }_{2} \mathrm{PF}_{6}$. J. Phys. Lett. (Paris) 1980, 41, L95-L98.

16. Jerome, D. The physics of organic superconductors. Science 1991, 252, 1509-1514.

17. Mori, H. Materials viewpoint of organic superconductors. J. Phys. Soc. Jpn. 2006, 75, 051003:1-051003:15.

18. Yu, W.; Zhang, F.; Zamborsky, F.; Alavi, B.; Baur, A.; Merlic, C.A.; Brown, S.E. Electron-lattice coupling and broken symmetries of molecular salt (TMTTF) ${ }_{2} \mathrm{SbF}_{6}$. Phys. Rev. B 2004, 70, 121101:1-121101:4.

19. Yoshimi, K.; Seo, H.; Ishibashi, S.; Brown, S.E. Tuning the magnetic dimensionality by charge ordering in the molecular TMTTF salts. Phys. Rev. Lett. 2012, 108, 096402:1-096402:5.

20. Seo, H.; Merino, J.; Yoshioka, H.; Ogata, M. Theoretical aspects of charge ordering in molecular conductors. J. Phys. Soc. Jpn. 2006, 75, 051009:1-051009:20. 
21. Fukui, K.; Yonezawa, T.; Shingu, H. A molecular orbital theory of reactivity in aromatic hydrocarbons. J. Chem. Phys. 1952, 20, 722-725.

22. Hoffman, R. An extended Hückel theory. I. Hydrocarbons. J. Chem. Phys. 1963, 39, 1397-1412.

23. Mori, T.; Kobayashi, A.; Sasaki, Y.; Kobayashi, H.; Saito, G.; Inokuchi, H. The intermolecular interaction of tetrathiafulvalene and bis(ethylenedithio)tetrathiafulvalene in organic metals. Calculation of orbital overlaps and models of energy-band structures. Bull. Chem. Soc. Jpn. 1984, 57, 627-633.

24. Chaikin, P. Field induced spin density waves. J. Phys. I Fr. 1996, 6, 1875-1898.

25. Osada, T.; Ohmichi, E. Magnetotransport and magnetic-field-induced density waves in low-dimensional layered conductors. J. Phys. Soc. Jpn. 2006, 75, 051006:1-051006:7.

26. Ovchinnikov, A.A. On the possibility of a metal-dielectric transition in one-dimensional systems with noninteger numbers of electrons per unit. Sov. Phys. JETP 1973, 37, 176-177.

27. Giamarchi, T. Theoretical framework for quasi-one dimensional systems. Chem. Rev. 2004, 104, 5037-5056.

28. Degiorgi, L.; Jerome, D. Transport and optics in quasi-one-dimensional organic conductors. J. Phys. Soc. Jpn. 2006, 75, 051004:1-051004:10.

29. Seo, H.; Fukuyama, H. Antiferromagnetic phases of one-dimensional quarter-filled organic conductors. J. Phys. Soc. Jpn. 1997, 66, 1249-1252.

30. Hiraki, K.; Kanoda, K. Wigner crystal type of charge ordering in an organic conductor with a quarter-filled band: (DI-DCNQI) 2 Ag. Phys. Rev. Lett. 1998, 80, 4737-4740.

31. Kakiuchi, T.; Wakabayashi, Y.; Sawa, H.; Itou, T.; Kanoda, K. Wigner crystallization in (DI-DCNQI $)_{2} \mathrm{Ag}$ detected by synchrotron radiation x-ray diffraction. Phys. Rev. Lett. 2007, 98, 066402:1-066402:4.

32. Seo, H.; Motome, Y. Spiral charge frustration in the molecular conductor (DI-DCNQI) ${ }_{2} \mathrm{Ag}$. Phys. Rev. Lett. 2009, 102, 196403:1-196403:4.

33. Chow, D.S.; Zamborszky, F.; Alavi, B.; Tantillo, D.J.; Baur, A.; Merlic, C.A.; Brown, S.E. Charge ordering in the TMTTF family of molecular conductors. Phys. Rev. Lett. 2000, 85, 1698-1701.

34. Nad, F.; Monceau, P.; Carcel, C.; Fabre, J.M. Dielectric response of the charge-induced correlated state in the quasi-one-dimensional conductor (TMTTF) ${ }_{2} \mathrm{PF}_{6}$. Phys. Rev. B 2000, 62, 1753-1756.

35. Monceau, P.; Nad, F.; Brazovskii, S. Ferroelectric Mott-Hubbard phase of organic (TMTTF) ${ }_{2} X$ conductors. Phys. Rev. Lett. 2001, 86, 4080-4083.

36. Nad, F.; Monceau, P. Dielectric response of the charge ordered state in quasi-one-dimensional organic conductors. J. Phys. Soc. Jpn. 2006, 75, 051005:1-051005:12.

37. Coulon, C.; Parkin, S.S.S.; Laversanne, R. Structureless transition and strong localization effects in bis-tetramethyltetrathiafulvalenium salts [(TMTTF $\left.)_{2} X\right]$. Phys. Rev. B 1985, 31, 3583-3587.

38. For $\mathrm{CO}$ in molecular conductors in general including studies in early stage by Verwey, Hubbard and others, see reference 20.

39. Clay, R.T.; Mazumdar, S.; Campbell, D.K. Bond-order and charge-density waves in the isotropic interacting two-dimensional quarter-filled band and insulating state proximate to organic superconductivity. Phys. Rev. B 2003, 67, 115121:1-115121:9.

40. Nakamura, T. Possible charge ordering patterns of the paramagnetic insulating states in (TMTTF) ${ }_{2}$ X. J. Phys. Soc. Jpn. 2003, 72, 213-216. 
41. Inagaki, S.; Fukuyama, H. Spin-peierls state vs. Neel state. II. Interchain exchange interaction. J. Phys. Soc. Jpn. 1983, 52, 3620-3629.

42. Zamborszky, F.; Yu, W.; Raas, W.; Brown, S.E.; Alavi, B.; Merlic, C.A.; Baur, A. Competition and coexistence of bond and charge orders in (TMTTF) ${ }_{2} \mathrm{AsF}_{6}$. Phys. Rev. B 2002, 66, 081103:1-081103:4.

43. Nakamura, T.; Furukawa, K.; Hara, T. Redistribution of charge in the proximity of spin-peierls transition: ${ }^{13} \mathrm{C}$ NMR investigation of $(\mathrm{TMTTF})_{2} \mathrm{PF}_{6}$. J. Phys. Soc. Jpn. 2007, 76, 064715:1-064715:5.

44. Itoi, M.; Hedo, M.; Uwatoko, Y.; Nakamura, T. Anomalously wide superconducting phase of one-dimensional organic conductor (TMTTF) $)_{2} \mathrm{SbF}_{6}$. J. Phys. Soc. Jpn. 2008, 77, 023701:1-023701:4.

45. Iwase, F.; Sugiura, K.; Furukawa, K.; Nakamura, T. ${ }^{13} \mathrm{C}$ NMR study of the magnetic properties of the quasi-one-dimensional conductor (TMTTF) ${ }_{2} \mathrm{SbF}_{6}$. Phys. Rev. B 2011, 84, 115140:1-115140:7.

46. Kino, H.; Fukuyama, H. Phase diagram of two-dimensional organic conductors: (BEDT-TTF) ${ }_{2} X$. J. Phys. Soc. Jpn. 1996, 65, 2158-2169.

47. Seo, H. Charge ordering in organic ET compounds. J. Phys. Soc. Jpn. 2000, 69, 805-820.

48. Seo, H.; Hotta, C.; Fukuyama, H. Toward systematic understanding of diversity of electronic properties in low-dimensional molecular solids. Chem. Rev. 2004, 104, 5005-5036.

49. Kanoda, K. Recent progress in NMR studies on organic conductors. Hyperfine Interactions 1997, 104, 235-249.

50. Kanoda, K. Metal-insulator transition in $\kappa-(\mathrm{ET})_{2} X$ and (DCNQI) ${ }_{2} \mathrm{M}$ : Two contrasting manifestation of electron correlation. J. Phys. Soc. Jpn. 2006, 75, 051007:1-051007:15.

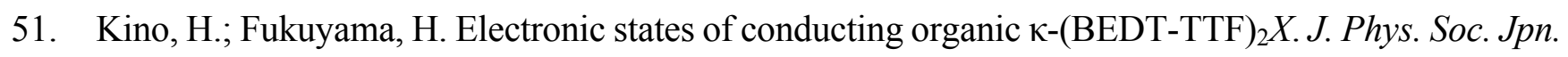
1995, 64, 2726-2729.

52. Shimizu, Y.; Miyagawa, K.; Kanoda, K.; Maesato, M.; Saito, G. Spin liquid state in an organic Mott insulator with a triangular lattice. Phys. Rev. Lett. 2003, 91, 107001:1-107001:4.

53. Anderson, P.W. Resonating valence bonds: A new kind of insulator? Mater. Res. Bull. 1973, 8, $153-160$.

54. Itou, T.; Oyamada, A.; Maegawa, S.; Tamura, M.; Kato, R. Quantum spin liquid in the spin-1/2 triangular antiferromagnet $\mathrm{EtMe}_{3} \mathrm{Sb}\left[\mathrm{Pd}(\mathrm{dmit})_{2}\right]_{2}$. Phys. Rev. B 2008, 77, 104413:1-104413:5.

55. Kato, R.; Itou, T. Molecular Quasi-triangular Lattice Antiferromagnets. In Understanding Quantum Phase Transitions; Lincoln, D.C., Ed.; CRC Press: Boca Raton, FL, USA, 2010; pp. 419-443.

56. Kanoda, K.; Kato, R. Mott physics in organic conductors with triangular lattices. Annu. Rev. Condens. Matter Phys. 2011, 2, 167-188.

57. Balants, L. Spin liquids in frustrated magnets. Nature 2010, 464, 199-208.

58. Abdel-Jawad, M.; Terasaki, I.; Sasaki, T.; Yoneyama, N.; Kobayashi, N.; Uesu, Y.; Hotta, C. Anomalous dielectric response in the dimer Mott insulator $\kappa-(\mathrm{BEDT}-\mathrm{TTF})_{2} \mathrm{Cu}_{2}(\mathrm{CN})_{3}$. Phys. Rev. $B$ 2010, 82, 125119:1-125119:5.

59. Li, H.; Clay, R.T.; Mazumdar, S. The paired-electron crystal in the two-dimensional frustrated quarter-filled band. J. Phys. Condens. Matter 2010, 22, 272201:1-272201:7.

60. Naka, M.; Ishihara, S. Electronic ferroelectricity in a dimer Mott insulator. J. Phys. Soc. Jpn. 2010, 79, 063707:1-063707:4. 
61. Gomi, H.; Imai, T.; Takahashi, A.; Aihara, M. Purely electronic terahertz polarization in dimer Mott insulators. Phys .Rev. 2010, B82, 035101:1-035101:4.

62. Hotta, C. Quantum electric dipoles in spin-liquid dimer Mott insulator $\kappa-\mathrm{ET}_{2} \mathrm{Cu}_{2}(\mathrm{CN})_{3}$. Phys. Rev. $B$ 2010, 82, 241104:1-241104:4.

63. Kishida, H. Possible interrelationship between dielectric responses and magnetic states has been elucidated by optical measurements. Nagoya University, Nagoya, Japan. Private communications, 2011.

64. Ejima, S.; Gebhard, F.; Nishimoto, S.; Ohta, Y. Phase diagram of $\mathrm{t}-\mathrm{U}_{-}-\mathrm{V}_{1}-\mathrm{V}_{2}$ model at quarter filling. Phys. Rev. B 2005, 72, 033101:1-033101:4.

65. Takahashi, T.; Nogami, Y.; Yakushi, K. Charge ordering in organic conductors. J. Phys. Soc. Jpn. 2006, 75, 051008:1-051008:17.

66. Tajima, N.; Sugawara, S.; Tamura, M.; Nishio, Y.; Kajita, K. Electronic phases in an organic conductor $\alpha$-(BEDT-TTF $)_{2} \mathrm{I}_{3}$ : Ultra narrow gap semiconductor, superconductor, metal, and charge-ordered insulator. J. Phys. Soc. Jpn. 2006, 75, 051010:1-051010:10.

67. Kino, H.; Fukuyama, H. On the phase transition of $\alpha-(\mathrm{ET})_{2} \mathrm{I}_{3}$. J. Phys. Soc. Jpn. 1995, 64, $1877-1880$.

68. Katayama, S.; Kobayashi, A.; Suzumura, Y. Pressure-induced zero-gap semiconducting state in organic conductor $\alpha$-(BEDT-TTF) ${ }_{2} \mathrm{I}_{3}$ salt. J. Phys. Soc. Jpn. 2006, 75, 054705:1-054705:6.

69. Kobayashi, A.; Katayama, S.; Noguchi, K.; Suzumura, Y. Superconductivity in charge ordered organic conductor $\alpha-(\mathrm{ET})_{2} \mathrm{I}_{3}$ salt. J. Phys. Soc. Jpn. 2004, 73, 3135-3148.

70. Ishibashi, S.; Tamura, M.; Kohyama, M.; Terakura, K. Ab Initio Electronic-Structure Calculations for $\alpha$-(BEDT-TTF) ${ }_{2} \mathrm{I}_{3}$. J. Phys. Soc. Jpn. 2006, 75, 015005:1-015005:2.

71. Kino, H.; Miyazaki, T. First-principles study of electronic structure in $\alpha$-(BEDT-TTF) $)_{2} \mathrm{I}_{3}$ at ambient pressure and with uniaxial strain. J. Phys. Soc. Jpn. 2006, 75, 034704:1-034704:8.

72. Kobayashi, A.; Katayama, S.; Suzumura, Y.; Fukuyama, H. Massless fermions in organic conductor. J. Phys. Soc. Jpn. 2007, 76, 034711:1-034711:6.

73. McClure, J.W. Diamagnetism of graphite. Phys. Rev. 1956, 104, 666-671.

74. Shon, N.H.; Ando, T. Quantum transport in two-dimensional graphite system. J. Phys .Soc. Jpn. 1998, 67, 2421-2429.

75. Novoselov, K.S.; Geim, A.K.; Morozov, S.V.; Jiang, D.; Katsnelson, M.I.; Grigorieva, I.V.; Dubonosand, S.V.; Firsov, A.A. Two-dimensional gas of massless Dirac fermions in graphene. Nature 2005, 438, 197-200.

76. Kubo, R.; Fukuyama, H. Interband Effects in Orbital Magnetism and Halll conductivity. In Proceedings of the 10th International Conference on Physics of Semiconductors, Cambridge, MA, USA, 17-21 August 1970; United States Atomic Energy Commission: Germantown, MD, USA, 1970; pp. 551-560.

77. Osada, T. Negative interlayer magnetoresistance and zero-mode Landau level in multilayer Dirac electron systems. J. Phys. Soc. Jpn. 2008, 77, 084711:1-084711:5.

78. McClure, J.W. Theory of diamagnetism of graphite. Phys. Rev. 1960, 199, 606-613.

79. Fukuyama, H. Anomalous orbital magnetism and Hall effect of massless fermions in two dimension. J. Phys. Soc. Jpn. 2007, 76, 043711:1-043711:3. 
80. Kobayashi, A.; Suzumura, Y.; Fukuyama, H. Hall effect and orbital diamagnetism in zerogap state of molecular conductor $\alpha$-(BEDT-TTF) $)_{2} \mathrm{I}_{3}$. J. Phys. Soc. Jpn. 2008, 77, 064718:1-064718:9.

81. Ando, T. Charged impurity scattering in graphenes: Effects of environmental screening, band gap, and AA stacking. J. Phys. Conf. Ser. 2011, 302, 012015:1-012015:8.

82. Tajima, N.; Kato, R.; Sugawara, S.; Nishio, Y.; Kajita, K. Interband effects of magnetic field on Hall conductivity in the multilayered massless Dirac fermion system $\alpha$-(BEDT-TTF) $)_{2} \mathrm{I}_{3}$. Phys. Rev. B 2012, 85, 033401:1-033401:4.

83. Kanoda, K. NMR Knight shift has indicated the reduction of effective density of states compared with the result of the band-structure calculations near the crossing points, while it is enhanced at higher energies. Tokyo University, Tokyo, Japan. Private communications, 2011.

84. Kotov, V.N.; Uchoa, B.; Pereira, V.M.; Guinea, F.; Castro, N.A.H. Electron-electron interactions in graphene: Current status and perspectives. Rev. Mod. Phys. 2012, in press.

85. Kobayashi, H.; Cui, H.; Kobayashi, A. Organic metals and superconductors based on BETS (BETS = Bis(ethylenedithio)tetraselenafulvalene). Chem. Rev. 2004, 104, 5265-5288.

86. Uji, S.; Brooks, J.S. Magnetic-field-induced superconductivity in organic conductors. J. Phys. Soc. Jpn. 2006, 75, 051014:1-051014:10.

87. Inabe, T.; Tajima, H. Phthalocyanines versatile components of molecular conductors. Chem. Rev. 2004, 104, 5503-5534.

88. Manriquez, J.M.; Gordon, T.Y.; McLean, R.S.; Epstein, A.J.; Miller, J.S. A room-temperature molecular/organic-based magnet. Science 1991, 252, 1415-1417.

89. Miller, J.S. Oliver Kahn lecture: Composition and structure of the V[TCNE $]_{\mathrm{x}}(\mathrm{TCNE}=$ tetracyanoethylene) room-temperature, organic-based magnet-A personal perspective. Polyhedron 2009, 28, 1596-1605.

90. Matuura, H.; Miyake, K.; Fukuyama, H. Theory of room temperature ferromagnet V(TCNE) $x$ $(1.5<x<2)$ : Role of hidden flat bands. J. Phys. Soc. Jpn. 2010, 79, 034712:1-034712:10.

91. Kato, R.; Kobayashi, H.; Kobayashi, A. Crystal and electronic structures of conductive anion-radical salts, $\left(2,5-R_{1} R_{2} \text {-DCNQI }\right)_{2} \mathrm{Cu}$ (DCNQI $=N$, $N$ '-dicyanoquinonediimine; $R_{1}, R_{2}=\mathrm{CH}_{3}$, $\left.\mathrm{CH}_{3} \mathrm{O}, \mathrm{Cl}, \mathrm{Br}\right)$. J. Am. Chem. Soc. 1989, 111, 5224-5232.

92. Kato, R. Conductive copper salts of 2,5-Disubstituted $N, N$ '-Dicyanobenzoquinonediimines (DCNQIs): Structural and physical properties. Bull. Chem. Soc. Jpn. 2000, 73, 515-534.

93. Tanaka, H.; Okano, Y.; Kobayashi, H.; Suzuki, W.; Kobayashi, A. A three-dimensional synthetic metallic crystal composed of single-component molecules. Science 2001, 291, 285-287.

94. Kobayashi, H.; Fujiwara, E.; Kobayashi, H. Single-component molecular metals with extended-TTF dithiolate ligands. Chem. Rev. 2004, 104, 5243-5264.

95. Tanaka, H.; Tokumoto, M.; Ishibashi, S.; Graf, D.; Choi, E.S.; Brooks, J.S.; Yasuzuka, S.; Okano, Y.; Kobayashi, H.; Kobayashi, A. Observation of three-dimensional Fermi surfaces in a single-component molecular metal, [Ni(tmdt) 2 ]. J. Am. Chem. Soc. 2004, 126, 10518-10519.

96. Rovira, C.; Novoa, J.J.; Mozos, J.L.; Ordejón, P.; Canadell, E. First-principles study of the neutral molecular metal Ni(tmdt)2. Phys. Rev. B 2002, 65, 081104:1-081104:4.

97. Ishibashi, S.; Tanaka, H.; Kohyama, M.; Tokumoto, M.; Kobayashi, A.; Kobayashi, H.; Terakura, K. Ab Initio electronic structure calculation for single-component molecular conductor $\mathrm{Au}(\mathrm{tmdt})_{2}$ $(\mathrm{tmdt}=$ Trimethylenetetrathiafulvalenedithiolate $)$. J. Phys. Soc. Jpn. 2005, 74, 843-846. 
98. Ishibashi, S.; Terakura, K.; Kobayashi, A. Electronic structures of single component molecular metals based on ab initio calculation. J. Phys. Soc. Jpn. 2008, 77, 024702:1-024702:7.

99. Yasuzuka, S.; Tanaka, H.; Tokumoto, M.; Graf, D.; Choi, E.S.; Brooks, J.S.; Kobayashi, H.; Kobayashi, A. Highly isotropic magnetoresistance in a single-component molecular metal [Ni(tmdt) 2 ]. J. Phys. Soc. Jpn. 2008, 77, 034709:1-034709:5.

100. Suzuki, W.; Fujiwara, E.; Kobayashi, A.; Fujishiro, Y.; Nishibori, E.; Takata, M.; Sakata, M.; Fujiwara, H.; Kobayashi, H. Highly conducting crystals based on single-component gold complexes with extended-TTF dithiolate ligands. J. Am. Chem. Soc. 2003, 125, 1486-1487.

101. Zhou, B.; Shimamura, M.; Fujiwara, E.; Kobayashi, A.; Higashi, T.; Nishibori, E.; Sakata, M.; Cui, H.B.; Takahashi, K.; Kobayashi, H. Magnetic transitions of single-component molecular metal $\left[\mathrm{Au}(\mathrm{tmdt})_{2}\right]$ and its alloy systems. J. Am. Chem. Soc. 2006, 128, 3872-3873.

102. Hara, Y.; Miyagawa, K.; Kanoda, K.; Shimamura, M.; Zhou, B.; Kobayashi, A.; Kobayashi, H. NMR evidence for antiferromagnetic transition in the single-component molecular conductor, [Au(tmdt) $)_{2}$ ] at 110 K. J. Phys. Soc. Jpn. 2008, 77, 053706:1-053706:4.

103. Seo, H.; Ishibashi, S.; Okano, Y.; Kobayashi, H.; Kobayashi, A.; Fukuyama, H.; Terakura, K. Single-component molecular metals as multiband $\pi-\mathrm{d}$ systems. J. Phys. Soc. Jpn 2008, 77, 023714:1-023714:4.

104. Zhou, B.; Yajima, H.; Kobayashi, A.; Okano, Y.; Tanaka, H.; Kumashiro, T.; Nishibori, E.; Sawa, H.; Kobayashi, H. Single-component molecular conductor $\left[\mathrm{Cu}(\mathrm{tmdt})_{2}\right]$ containing an antiferromagnetic Heisenberg chain. Inorg. Chem. 2010, 49, 6740-6747.

105. Tsuneyuki, S.; Kobori, T.; Akagi, K.; Sodeyama, K.; Terakura, K.; Fukuyama, H. Molecular orbital calculation of biomolecules with fragment molecular orbitals. Chem. Phys. Lett. 2009, 476, 104-108.

106. Nishioka, H.; Ando, K. Electronic coupling calculation and pathway analysis of electron transfer reaction using ab initio fragment-based method. I. FMO-LCMO approach. J. Chem. Phys. 2011, 134, 204109:1-204109:12.

107. Harada, Y.; Taguchi, M.; Miyajima, Y.; Tokushima, T.; Horikawa, Y.; Chainani, A.; Shiro, Y.; Senba, Y.; Ohashi, H.; Fukuyama, H.; et al. Ligand energy controls the Heme-Fe valence in aqueous myoglobins. J. Phys. Soc. Jpn. 2009, 78, 044802:1-044802:5.

108. Day, P.; Smith, D.W.; Williams, R.J.P. Crystal spectra of a Heme and some Heme-protein complexes. Biochemistry 1967, 6, 1563-1566.

109. Day, P.; Smith, D.W.; Williams, R.J.P. Crystal spectra of some ferric hemoproteins. Biochemistry 1967, 6, 3747-3750.

110. Fukuyama, H. Physics of molecular conductors. J. Phys. Soc. Jpn. 2006, 75, 051001:1-051001:7.

111. Fukuyama, H. Trends of Condensed Matter Science: A Personal View. In Frontiers in Materials Research; Fujikawa, Y., Nakajima, K., Sakurai, T., Eds.; Springer: Berlin/Heidelberg, Geramny, 2008; pp. 11-28.

(C) 2012 by the authors; licensee MDPI, Basel, Switzerland. This article is an open access article distributed under the terms and conditions of the Creative Commons Attribution license (http://creativecommons.org/licenses/by/3.0/). 